\title{
Astaxanthin as a Medical Food
}

\author{
Eiji Yamashita
}

Department of Research \& Development, Medical Nutrition Division, AstaReal Co., Ltd., Tokyo, Japan

Corresponding author: Eiji Yamashita, 2-6-3-12F, Shibakoen, Minato-ku, Tokyo 105-0011, Japan

Submission date: April 28, 2013; Acceptance date: July 3, 2013; Publication date: July 3, 2013

\section{ABSTRACT:}

Astaxanthin is a red pigment that belongs to the carotenoid family like $\beta$-carotene. And it's found in seafood such as crustaceans: shrimp and crabs and fish: salmon and sea bream. Recently, astaxanthin has been reported to have antioxidant activity up to 100 times more potent than that of vitamin $\mathrm{E}$ against lipid peroxidation and about 40 times more potent than that of $\beta$-carotene on singlet oxygen quenching. Astaxanthin does not show any pro-oxidant activity and its main sight of action is on/in the cell membrane. Various important benefits to date have suggested for human health such as immunomodulation, anti-stress, anti-inflammation, LDL cholesterol oxidation suppression, enhanced skin health, improved semen quality, attenuating eye fatigue, sport performance and endurance, limiting exercised induced muscle damage, suppressing the development of life-style related diseases such as obesity, atherosclerosis, diabetes, hyperlipidemia and hypertension. Nowadays, the research and demand for natural astaxanthin in human health application are explosively growing worldwide. Especially, the clinicians use the astaxanthin extracted from the microalgae, Haematotoccus pluvialis, as an add-on supplementation for the patients who are unsatisfied with the current medications or who can't receive any medications because of their serious symptom. For example, the treatment enhances their daily activity levels or QOL in heart failure or benign prostatic hypertrophy/lower urinary tract symptom patients. Other studies and trials are under way on chronic diseases such as nonalcoholic steatohepatitis, diabetes and CVD. We may call astaxanthin "a medical food" in the near future.

Keywords: astaxanthin, medical food, Haematococcus, add-on supplementation

\section{BACKGROUND:}

Astaxanthin is widely and naturally distributed in marine organisms including crustaceans such as shrimps and crabs and fish such as salmon and sea bream. In fact, it is one of the oldest carotenoids isolated and identified from lobster, Astacus gammarus [1]. Astaxanthin was first 
commercially used for pigmentation only in the aquaculture industry. Later in 1991, when the biological activity of potent antioxidative property was reported [2], astaxanthin as a food supplement started gaining acceptance. Nowadays, the research and demand for natural astaxanthin in human health application are explosively growing worldwide. In this article the underlying basis to astaxanthin's bioactivity and health promotional effects of natural astaxanthin extracted from the microalgae, Haematotoccus pluvialis, are reviewed. And the practical medical applications of natural astaxanthin are disclosed with the cases and reports by the clinicians using the astaxanthin as an add-on supplementation for the patients who are unsatisfied with the current medications or who can't receive any medications because of their serious symptom.

Uunderlying basis to astaxanthin's bioactivity: Astaxanthin is a totally unique antioxidant, because it possesses three novel distinctions at once.

Powerful antioxidant: The inhibitory activity of astaxanthin on the peroxyl radical mediated lipid peroxidation was more than 100 times greater than that of $\alpha$-tocopherol in the homogenate of rat mitochondria [2]. Among twenty seven common hydrophilic and lipophilic antioxidants such as polyphenols, toco-pherols, carotenoids, ascorbic acid, coenzyme Q10 and $\alpha$-lipoic acid astaxanthin showed the strongest singlet oxygen $\left({ }^{1} \mathrm{O}_{2}\right)$ quenching activity under the same test condition of the chemilu-minescence detection system for direct ${ }^{1} \mathrm{O}_{2}$ counting using the thermodissociable endoperoxides of 1,4-dimethylnaphthalene as ${ }^{1} \mathrm{O}_{2}$ generator in DMF : $\mathrm{CDCl}_{3}$ (9 : 1) [3]. Hydroxyl radical scavenging ability of astaxanthin encapsulated in liposomes was more potent than that of $\alpha$-tocopherol [4].

Safe antioxidant: Martin divided seventeen investigated carotenoids into three classes ; i) without significant antioxidative properties, ii) anti- and pro-oxidants and iii) pure anti-oxidants. Astaxanthin was classified as "pure anti-oxidants" not possessing any pro-oxidative properties like $\beta$-carotene and lycopene [5]. Non-polar carotenoids such as lycopene and $\beta$-carotene disordered the membrane bilayer enriched with polyunsaturated fatty acids and showed a potent pro-oxidant effect (>85\% increase in lipid hydroperoxide (LOOH) levels) while astaxanthin preserved membrane structure and exhibited significant antioxidant activity $(40 \%$ decrease in LOOH levels) [6]. The photostability of the three carotenoids in the human dermal fibroblasts was astaxanthin $>$ canthaxanthin $>>\beta$-carotene. Only astaxantin efficiently abrogated the apoptotic response to UVA. $\beta$-Carotene dose-dependently induced caspase- 3 activity following UVA exposure [7].

Superior position in cell membrane: Astaxanthin traps radicals not only at the conjugated polyene chain but also in the terminal ring moiety, in which the hydrogen atom at the $\mathrm{C} 3$ methine is suggested to be a radical trapping site. Owing to the equivalent amounts of the hydrophobic intramolecular hydrogen-bonded ring and intermolecular hydrogen bonding with phospholipid polar heads, and the interconversion between the two hydrogen bond formations, the terminal ring of astaxanthin is able to scavenge radicals both at the surface and in the interior of the phospholipid membrane, although its unsaturated polyene chain trapped radicals only in the 
membrane [8]. Figure 1 shows astaxanthin's unique ability to span through the double layer cell membrane. $\beta$-carotene and vitamin $\mathrm{C}$ only reside inside and outside the lipid bilayer membrane respectively. The astaxanthin molecule is exposed both in- and outside of the cell giving better overall protection.

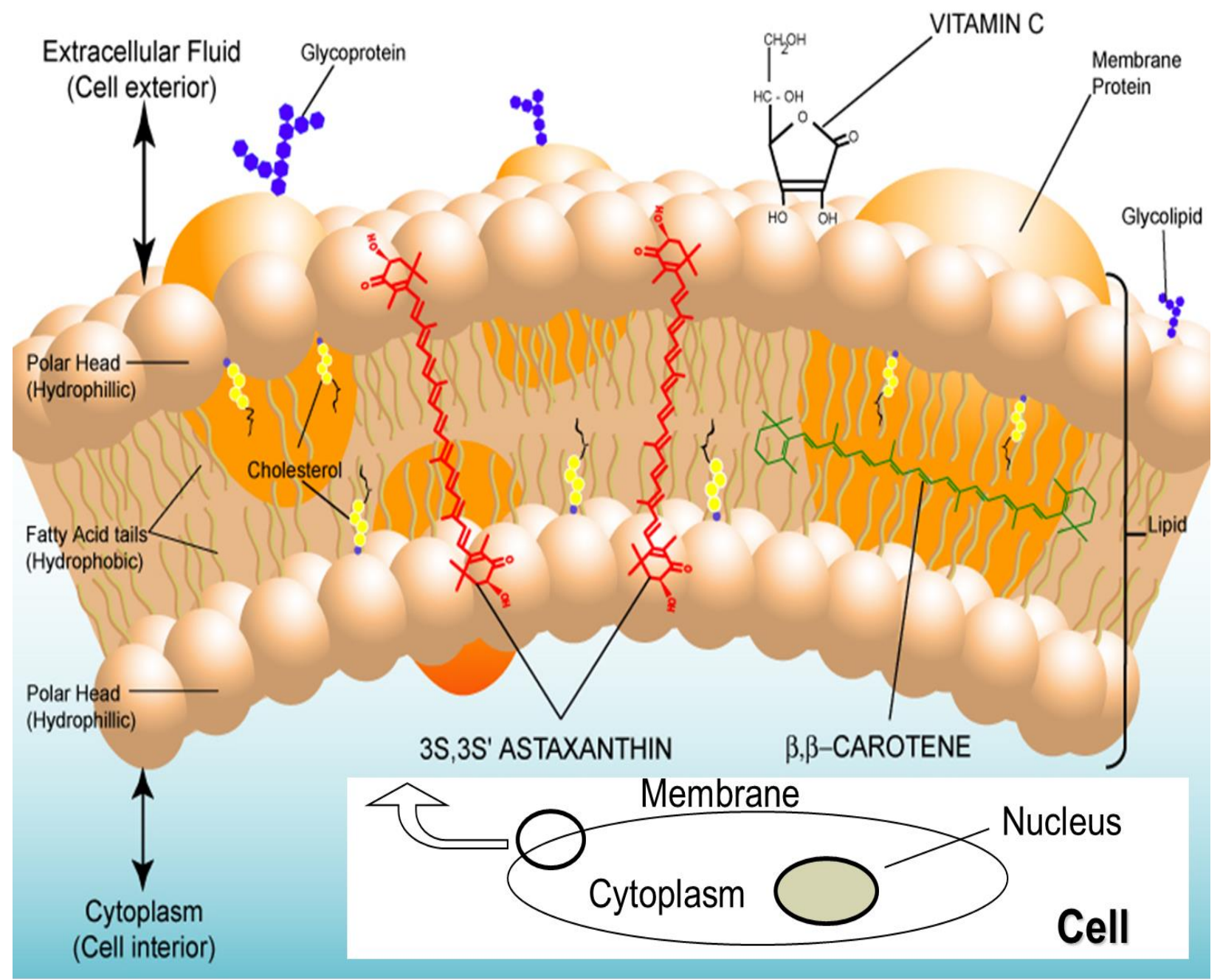

Figure 1. Superior position in cell membrane. No other antioxidants possess the three unique characters at once. This should be associated with its potent bioactivity.

Health promotional effects of natural astaxanthin: Numerous studies have shown that astaxanthin has potential health-promoting effects in the prevention and treatment of various diseases, such as cancers, chronic inflammatory diseases, metabolic syndrome, diabetes, diabetic nephropathy, cardiovascular diseases, gastrointestinal diseases, liver diseases, neurodegenerative diseases, eye diseases, skin diseases, exercise-induced fatigue, male infertility as reviewed by Yuan [9]. It has been proven in over 65 clinical studies, featured in over 300 peer-reviewed publications. Figure 2 shows the main benefits for human health. "Eye fatigue relieve" [10], "skin aging defense" (anti-photoaging) [11] and "muscle resilience" (sports performance enhancement) $[12,13]$ have been most clinically substantiated. 


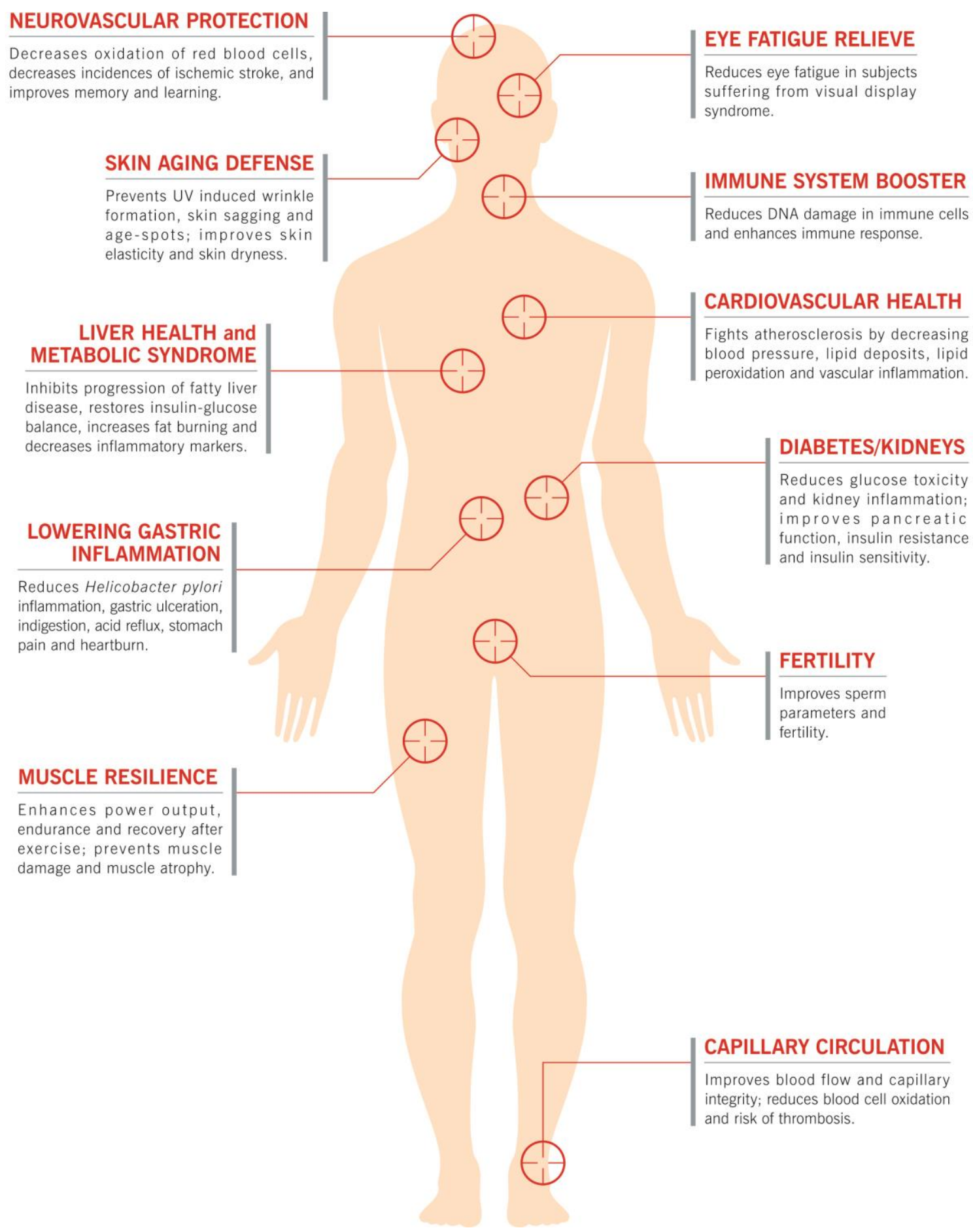

CAstraReal 2012

Figure 2. Health promotional effects of astaxanthin

Practical medical applications of natural astaxanthin: Mostly two soft gel capsules of the dietary supplement containing $12 \mathrm{mg}$ of AstaREAL® astaxanthin extracted from the microalgae, Haematotoccus pluvialis were used by the clinicians. Add-on supplementations of AstaREAL® astaxanthin dramatically exhibited improvements in the patients who were unsatisfied with the 
current medications or who couldn't receive any medications because of their serious symptom. There were the cases that the administration improved the cardiac function in the heart failure patients who is difficult to control with drug therapy and in the patients whose activity levels were reduced because of chronic heart failure resulting in enhancing their daily activity levels and QOL. The add-on effects of astaxanthin for treatment of benign prostatic hypertrophy/lower urinary tract symptom (BPH/LUTS) were reported as an open-label preliminary study. A total of thirty patients who had been treated with a1-blockers for more than 12 weeks and still had LUTS intook the astaxanthin for eight weeks. The subjective symptoms and objective voiding parameters were improved including QOL. Other studies and trials are under way on chronic diseases such as non-alcoholic steatohepatitis, diabetes and CVD as well as infertility, atopic dermatitis and androgenetic alopecia.

\section{CONCLUSION:}

A practical medical application of astaxanthin from Haematococcus will expand into medical institution worldwide not only into the consumer space. We may call astaxanthin "a medical food" at the moment.

\section{REFERENCES:}

1. Kuhn R, Sorensen NA The coloring matters of the lobster (Astacus gammarus L.). Z Angew Chem 1938; 51: 465-466.

2. Miki W. Biological functions and activities of animal carotenoids. Pure Appl Chem 1991; 63: 141-146.

3. Nishida Y, Yamashita E, Miki W. Quenching activities of common hydrophilic and lipo-philic antioxidants against singlet oxygen using chemiluminescence detection system. Car Sci 2007; 11: 16-20.

4. Hama S, Uenishi S, Yamada A, Ohgita T, Tsuchiya H, Yamashita E, Kogure K. Scavenging of hydroxyl radicals in aqueous solution by astaxanthin encapsulated in liposomes. Biol. Pharm. Bull. 2012; 35(12): 2238-2242.

5. Martin HD, Ruck C, Schmidt M, Sell S, Beutner S, Mayer B, Walsh R. Chemistry of carote-noid oxidation and free radical reactions. Pure \& Appl Chem 1999; 71: 2253 2262.

6. McNulty HP, Byun J, Lockwood SF, Jacob RF, Mason RP. Differential effects of carotenoids on lipid peroxidation due to membrane interactions: X-ray diffraction analysis. Biochim Biophys Acta. 2007; 1768: 167-174.

7. Camera E, Mastrofrancesco A, Fabbri C, Daubrawa F, Picardo M, Sies H, Stah W. Astaxanthin, canthaxanthin and $\beta$-carotene differently affect UVA-induced oxidative damage and expression of oxidative stress-responsive enzymes. Exp Dermatol. 2009; 18(3): 222-231.

8. Goto S, Kogure K, Abe K, Kimata K, Kitahama K, Yamashita E, Terada H. Efficient radical trapping at the surface and inside the phospholipid membrane is responsible for highly potent antiperoxidative activity of the carotenoid astaxanthin. Biochim Biophys Acta. 2001; 1515: 251-258. 
9. Yuan JP, Peng J, Yin K, Wang JH. Potential health-promoting effects of astaxanthin: A high-value carotenoid mostly from microalgae. Mol Nutr Food Res. 2010; 55(1): 150165.

10. Yamashita E. Eye fatigue (asthenopia) relief by astaxanthin. Anti-Aging Therap. 2009; 11: 361-379.

11. Tominaga K, Hongo N, Karato M, Yamashita E. Cosmetic benefits of astaxanthin on humans subjects. Acta Biochim Polonoca. 2012; 59: 43-47.

12. Yamashita E. Astaxanthin and sports performance (in Japanese). FOOD Style 21. 2011; 15(3): 36-38.

13. Earnest CP, Lupo M, White KM, Church TS. Effect of astaxanthin on cycling time trial performance. Int J Sports Med. 2011; 32(11): 882-888. 